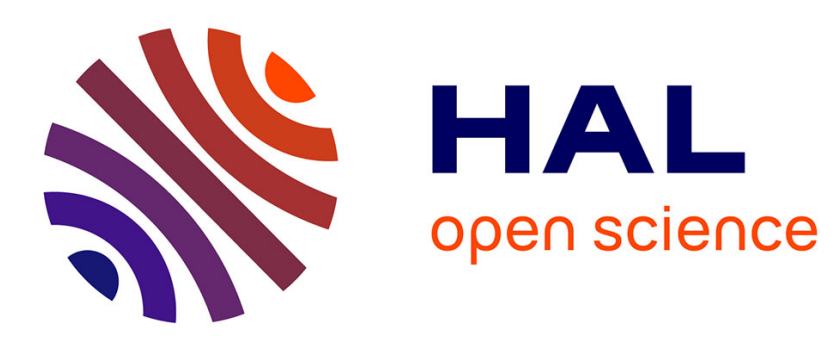

\title{
Precise damping and stiffness extraction in acoustic driven cantilever in liquid
}

Abdelhamid Maali, Rodolphe Boisgard

\section{To cite this version:}

Abdelhamid Maali, Rodolphe Boisgard. Precise damping and stiffness extraction in acoustic driven cantilever in liquid. Journal of Applied Physics, 2013, 114 (14), pp.144302 (1-5). 10.1063/1.4824459 . hal-00910164

\section{HAL Id: hal-00910164 \\ https://hal.science/hal-00910164}

Submitted on 19 Feb 2016

HAL is a multi-disciplinary open access archive for the deposit and dissemination of scientific research documents, whether they are published or not. The documents may come from teaching and research institutions in France or abroad, or from public or private research centers.
L'archive ouverte pluridisciplinaire HAL, est destinée au dépôt et à la diffusion de documents scientifiques de niveau recherche, publiés ou non, émanant des établissements d'enseignement et de recherche français ou étrangers, des laboratoires publics ou privés.

\section{(1)(1) $\$(0)$}

Distributed under a Creative Commons Attribution - NonCommercial - ShareAlikel 4.0 


\title{
Precise damping and stiffness extraction in acoustic driven cantilever in liquid
}

\author{
Abdelhamid Maalia) and Rodolphe Boisgard \\ Université Bordeaux 1, LOMA UMR 5798 CNRS, 351 Cours de la Libération, F 33400 Talence, France
}

\begin{abstract}
In this paper, we first explain how to extract accurately the driving force acting on the acoustic driven atomic force microscope cantilever in liquid from the measured resonance curve. We present a model that includes the driving force to extract precisely the damping and the stiffness of the tip sample interaction. The model is validated by an experimental test based on two independent methods to measure the hydrodynamic drag coefficient of a sphere moving perpendicular to flat surface.
\end{abstract}

\section{INTRODUCTION}

The atomic force microscope (AFM) provides a sensitive sensor ${ }^{1-8}$ to investigate materials properties at nanometer scale. The surface topography and various properties of organic and inorganic surfaces have been obtained with high resolution in vacuum, ${ }^{4}$ in air and in liquid. ${ }^{3-5}$ The dynamic AFM is widely used to image soft material especially when the imaged objects are isolated. The most popular and less expensive method to put in oscillation, the AFM cantilever is the acoustic excitation method. A piezoelectric actuator is used to excite the vibration of the cantilever base. For a cantilever having high quality factor, as is the case in vacuum and air, the displacement of the cantilever base is negligible compared to the deflection of the tip when the cantilever is excited close to the resonance.

For a cantilever acoustically excited in liquid medium, it has been pointed out that the cantilever oscillation amplitude is comparable to the base displacement. ${ }^{9-14}$ Thus, the whole motion of the tip is the sum of the base displacement and the deflection oscillation amplitude of the cantilever. ${ }^{9-14}$ Furthermore, the piezo actuator transmits vibration to the surrounding fluid, which then applies an additional force to the cantilever. This makes a challenge and complicates the extraction of the interaction properties: force, damping, and stiffness.

In this work, we first explain how to extract accurately the driving force acting on the cantilever in liquid from the measured resonance curve. Then, we present a model that includes the driving force to extract precisely the damping and the stiffness of the tip sample interaction.

\section{THEORETICAL MODELING}

Fig. 1 shows the model for a cantilever excited acoustically by a piezo actuator. For a cantilever excited at frequency $\omega$, the deflection of the tip $X(t)$ is described by the oscillator model equation ${ }^{10,12,14}$

$$
m^{*} \ddot{X}+\gamma_{\text {bulk }} \dot{X}+k_{c} X=F_{\text {drive }},
$$

\footnotetext{
a) Author to whom correspondence should be addressed. Electronic mail: a.maali@loma.u bordeaux1.fr
}

where $m^{*}$ is the effective mass of the cantilever, $k_{c}$ is the cantilever force constant, and $\gamma_{\text {bulk }}$ is the bulk damping coefficient far from the surface.

In the ideal situation, the driving force in liquid medium $F_{\text {drive }}$ induced by the displacement of the cantilever base can be calculated using the Euler-Bernoulli beam theory. ${ }^{12-14}$ The analytical expression of the driving force is

$$
F_{\text {drive }}=\left(m^{*} \omega^{2}-j \omega \gamma_{\text {bulk }}\right) \beta A_{b} e^{j \omega t},
$$

where $\beta=\frac{\int_{0}^{L} \Psi(x) d x}{\int_{0}^{L} \Psi^{2}(x) d x} \approx 1.565, \Psi(x)$ is the eigenmode shape of the first mode and $L$ is the cantilever beam length. $A_{b}$ is the amplitude of the base displacement. In our previous paper, ${ }^{10}$ the coefficient $\beta$ was omitted because the driving force was calculated using a point-mass-oscillator model.

In a real experiment, part of the cantilever excitation comes from the base vibration, and the other part comes from the acoustic wave that propagates from the piezo-actuator through the fluid. ${ }^{14}$ The total excitation is the sum of these two parts of excitation. Accurate determination of the contribution of the acoustic wave propagation is complicated because it may depend on the shape and the fixation of the cantilever on the holder. We show below that it is much easier to measure the whole driving force for a given cantilever in liquid.

Assuming a general expression for the total driving force in the form

$$
F_{\text {drive }}=\left(F_{1}+j F_{2}\right) e^{j \omega t},
$$

where $F_{1}$ is the term in the driving force that is in phase with acoustic vibration of the base and $F_{2}$, the term that is in phase quadrature.

By substituting expression (3) in (1) and using $X(t)=A_{\text {free }} e^{j\left(\omega t+\varphi_{\text {free }}\right)}$, we get

$$
\left.\frac{F_{1}}{k_{c}}=A_{\text {free }}\left[1-\left(\frac{\omega}{\omega_{0}}\right)^{2}\right) \cos \left(\varphi_{\text {free }}\right)-\left(\frac{\omega}{\omega_{0} Q}\right) \sin \left(\varphi_{\text {free }}\right)\right] \text {, }
$$

$$
\left.\frac{F_{2}}{k_{c}}=A_{\text {free }}\left[1-\left(\frac{\omega}{\omega_{0}}\right)^{2}\right) \sin \left(\varphi_{\text {free }}\right)+\left(\frac{\omega}{\omega_{0} Q}\right) \cos \left(\varphi_{\text {free }}\right)\right] \text {. }
$$




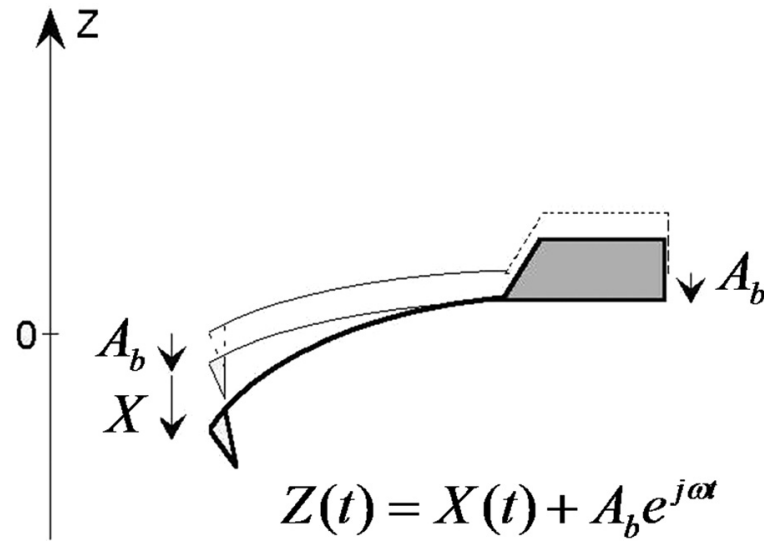

FIG. 1. Schematic diagram of the cantilever configuration. The instantane ous tip position $Z$ is the sum of the measured deflection $X$ and the base displacement: $Z \quad X+A_{d} e^{(j \omega t)}$.

$A_{\text {free }}$ and $\varphi_{\text {free }}$ are, respectively, the free amplitude and phase of the cantilever measured far from the surface. $\omega_{0}$ is the angular resonance frequency of the cantilever far from the surface $\left(\omega_{0}=\sqrt{\frac{k_{c}}{m^{*}}}\right)$ and $Q$ is the bulk quality factor of the cantilever $\left(Q=\frac{m^{*} \omega_{0}}{\gamma_{b u l k}}\right)$. The measurement of the resonance curve (amplitude and phase versus driving frequency) provides the driving force components $F_{1}$ and $F_{2}$ by the mean of Eqs. (4a) and (4b).

When the tip interacts with a sample, we have to add to the second term of Eq. (1) the interaction force. For a cantilever oscillating with small amplitude compared to the range of the interaction length, the instantaneous interaction force can be linearised. It has two contributions; one is due to the conservative term $\left(-k_{\text {int }} Z\right)$ and the other one is due to the dissipative term $\left(-\gamma_{\text {int }} \dot{Z}\right)$. Where $k_{\text {int }}, \gamma_{\text {int }}$ are, respectively, the interaction stiffness and damping, including pure tip sample interaction and fluid added mass and added damping close to the surface (see Appendix). The total tip displacement $Z$ is given by: $Z=X(t)+A_{b} e^{j \omega t}$.

Equation (1) becomes

$$
\begin{aligned}
& m^{*} \ddot{X}+\left(\gamma_{\text {bulk }}+\gamma_{\text {int }}\right) \dot{X}+\left(k_{c}+k_{\text {int }}\right) X \\
& \quad=\left(F_{1}+j F_{2}\right) e^{j \omega t}-\left(k_{\text {int }}+j \omega \gamma_{\text {int }}\right) A_{b} e^{j \omega t} .
\end{aligned}
$$

Under the study condition $X(t)=A e^{j(\omega t+\varphi)}$, we get

$$
\begin{aligned}
k_{\mathrm{int}}+j \omega \gamma_{\mathrm{int}} & =\frac{F_{1}+j F_{2}+\left(m^{*} \omega^{2}-k_{c}-j \omega \gamma_{b u l k}\right) A e^{j \varphi}}{A e^{j \varphi}+A_{b}} \\
= & \frac{F_{1}+j F_{2}+\left[\left(\frac{\omega}{\omega_{0}}\right)^{2}-1-j \frac{\omega}{\omega_{0} Q}\right] k_{c} A e^{j \varphi}}{A e^{j \varphi}+A_{b}} .
\end{aligned}
$$

Separating the real and the imaginary part of the previous equation yield the desired expressions for the damping and the stiffness of the tip-sample interaction.

$$
\begin{gathered}
\frac{\omega \gamma_{\text {int }}}{k_{c}}=\frac{-\frac{A}{A_{b}} \frac{F_{1}}{k_{c} A_{b}} \sin (\varphi)+\frac{F_{2}}{k_{c} A_{b}}\left(1+\frac{A}{A_{b}} \cos (\varphi)\right)-\frac{A}{A_{b}} \sin (\varphi)\left(1-\frac{\omega^{2}}{\omega_{0}^{2}}\right)-\frac{A}{A_{b}} \frac{\omega}{\omega_{0} Q}\left(\frac{A}{A_{b}}+\cos (\varphi)\right)}{1+\left(\frac{A}{A_{b}}\right)^{2}+2 \frac{A}{A_{b}} \cos (\varphi)}, \\
\frac{k_{\text {int }}}{k_{c}}=-1+\frac{\omega^{2}}{\omega_{0}^{2}}+\frac{\frac{A}{A_{b}} \frac{F_{1}}{k_{c} A_{b}} \cos (\varphi)+\frac{A}{A_{b}} \frac{F_{2}}{k_{c} A_{b}} \sin (\varphi)+\left(1+\frac{A}{A_{b}} \cos (\varphi)\right)\left(1-\frac{\omega^{2}}{\omega_{0}^{2}}\right)+\frac{F_{1}}{k_{c} A_{b}}+\frac{A}{A_{b}} \frac{\omega}{\omega_{0} Q} \sin (\varphi)}{1+\left(\frac{A}{A_{b}}\right)^{2}+2 \frac{A}{A_{b}} \cos (\varphi)} .
\end{gathered}
$$

In practice, the knowledge of the driving forces $F_{1}$ and $F_{2}$, the quality factor $Q$, the resonance frequency $\omega_{0}$ and the base displacement amplitude $A_{b}$ allows accurate extraction of $k_{\text {int }}, \gamma_{\text {int }}$.

To check the validity of our model, we study the motion of a tip ended by a sphere. We focus our test of the measurement of $\gamma_{\text {int }}$ since we have an alternative method to determine it. The hydrodynamic drag force that acts on a sphere moving perpendicular to a flat substrate is given by: $:^{7,15-17}$ $F_{h}=\frac{6 \pi \eta R^{2}}{D} V$, where $R$ and $\eta$ are the radius of the sphere and the dynamic viscosity of the fluid, $D$ is the distance between the surface and the sphere, and $V$ is the velocity of the sphere. In the above equation, we have assumed no boundary slip for the liquid on both surfaces; this assumption is valid in our experiment because we have used a hydrophilic glass sphere and mica substrate. For a sphere approaching to the substrate with a constant velocity (drainage experiment), the hydrodynamic drag coefficient that we can measure: $\gamma_{h}=\frac{F_{h}}{V}=\frac{6 \pi \eta R^{2}}{D}$ should be equal to the interaction-damping coefficient $\gamma_{\text {int }}$ Eq. (7b).

\section{RESULT AND DISCUSSION}

The experiment was performed using an AFM (Bruker, Bioscope) equipped with a liquid cell (DTFML-DD-HE) that allows both tapping and contact modes in liquid. The cantilevers used are silicon nitride rectangular cantilever ORC8, purchased from Bruker AFM Probes (width $=40 \mu \mathrm{m}$ 
length $=200 \mu \mathrm{m}$ thickness $=0.8 \mu \mathrm{m})$. We have used spherical borosilicate particle (GL0186B/45-53, MO-Sci Corporation) with a diameter of $102.8 \mu \mathrm{m}$. The sphere was glued to the end of the cantilever using epoxy (Araldite, Bostik, Coubert) (Fig. 2). The substrate is fixed on multi-axis piezo-stage (NanoT series, Mad City Labs) that allows a large displacement (Up to $50 \mu \mathrm{m}$ for applied voltage of $10 \mathrm{v}$ ) with a high accuracy under closed loop control. The amplitude and phase of the tip were measured using a lock-in amplifier (Signal Recovery Model 7280) with an integration time of $100 \mathrm{~ms}$.

First, we perform a drainage experiment in order to measure the hydrodynamic drag coefficient and to calibrate in situ the cantilever with the sphere attached to its end. This step was performed in contact mode (the acoustic excitation was switched off). The force was generated by the rapid approach (or withdraw) of the mica substrate to the sphere using the piezo-stage..$^{715-17}$ To analyze the recorded data, in this part, we have followed the procedure given by Honig and Ducker ${ }^{16}$ and Zhu et al. ${ }^{17}$ The measured deflection for the approach and retract is shown in Fig. 3(a).

The deflection of the cantilever (def) is related to the force $\left(\mathrm{F}_{\mathrm{h}}\right)$ by: $\frac{d e f}{V}=\frac{F_{h}}{k_{c} V}=\frac{6 \pi \eta R^{2}}{k_{c} D}$. The actual velocity of the approach (retract) was obtained from the time derivative of the distance. ${ }^{16}$ From the fit shown in Fig. 3(b), we obtain $\frac{6 \pi \eta R^{2}}{k_{c}}=0.261 \mathrm{~nm} \cdot \mathrm{s}$, using the numerical values of the sphere radius $R=51.4 \mu \mathrm{m}$ and the water viscosity $\eta=0.89 \mathrm{mPa} \mathrm{s}$,
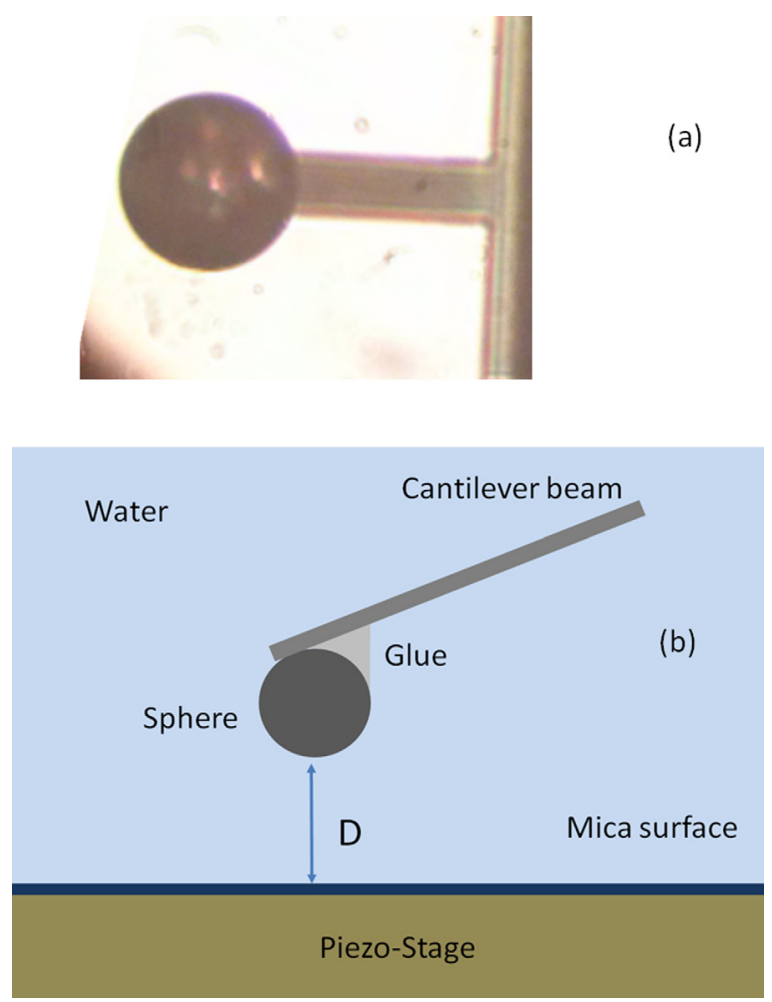

FIG. 2. (a) optical image of the sphere attached to end of the cantilever. (b) Schematic diagram of the experimental setup used to test the model. A glass sphere with a diameter of $102.8 \mu \mathrm{m}$ was attached at the end of silicon nitride cantilever (width $40 \mu \mathrm{m}$, length $200 \mu \mathrm{m}$, thickness $0.8 \mu \mathrm{m}$ ). The sub strate (mica surface) is fixed on piezo stage that allows a large displacement (Up to $50 \mu \mathrm{m}$ for applied voltage of $10 \mathrm{v}$ ).
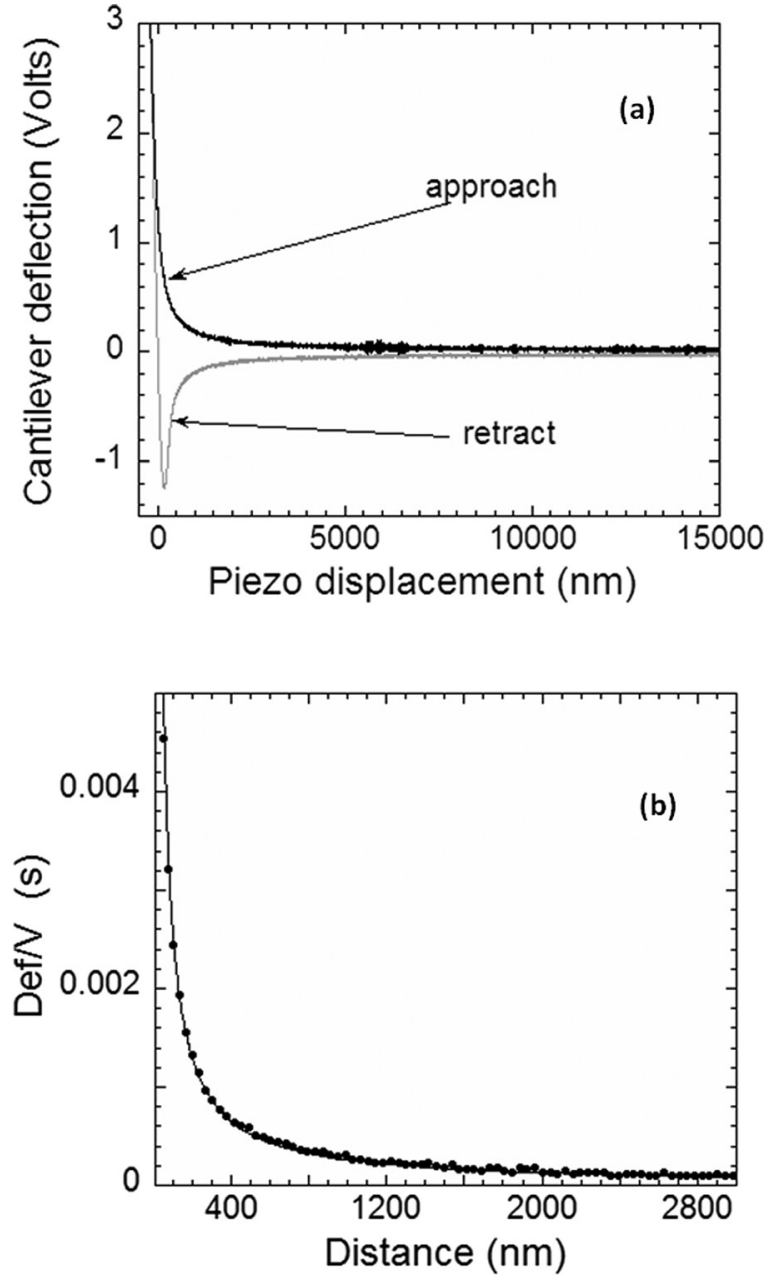

FIG. 3. (a) Measured cantilever deflection using a contact mode. The piezo stage displaces the substrate to the sphere with a velocity of $40 \mu \mathrm{m} / \mathrm{s}$. (b) The def/V and the fitting curve using equation $\frac{d e f}{V} \frac{6 \pi \eta R^{2}}{k_{c} D}$. From the fit

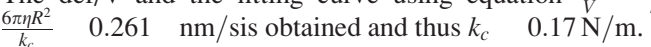

we get the stiffness value $k_{c}=0.17 \mathrm{~N} / \mathrm{m}$. Note that the curve plotted in Fig. 3(b) is no more than the hydrodynamic drag coefficient divided by the stiffness $\frac{\gamma_{h}}{k_{c}}$.

Now, we perform experiment in dynamic mode to measure the hydrodynamic interaction damping $\gamma_{\text {int }}$. First, we need to measure the exact value of the driving force acting on the tip. This force is determined from the resonance curves of the cantilever in bulk free from any interaction.

Fig. 4 shows the resonance curves of the cantilever with the sphere attached measured by thermal response and acoustic excitations. The fit of the thermal response curve (Fig. 4(a)) using the standard harmonic oscillator model allows to extract the resonance frequency $\omega_{0}$ and the quality factor $Q$ of the cantilever far from any interaction. Then, we use Eqs. (4a) and (4b) to calculate the driving force $F_{1}$ and $F_{2}$ shown in Fig. 4(c).

Then, we vibrate the cantilever at a given frequency and measure the amplitude and phase of the tip as the substrate approaches the sphere glued at the end of the cantilever with a very low rate. On Fig. 5(a) is shown the amplitude of the cantilever versus the distance for different frequencies of vibration. Close to the contact position, the damping is 

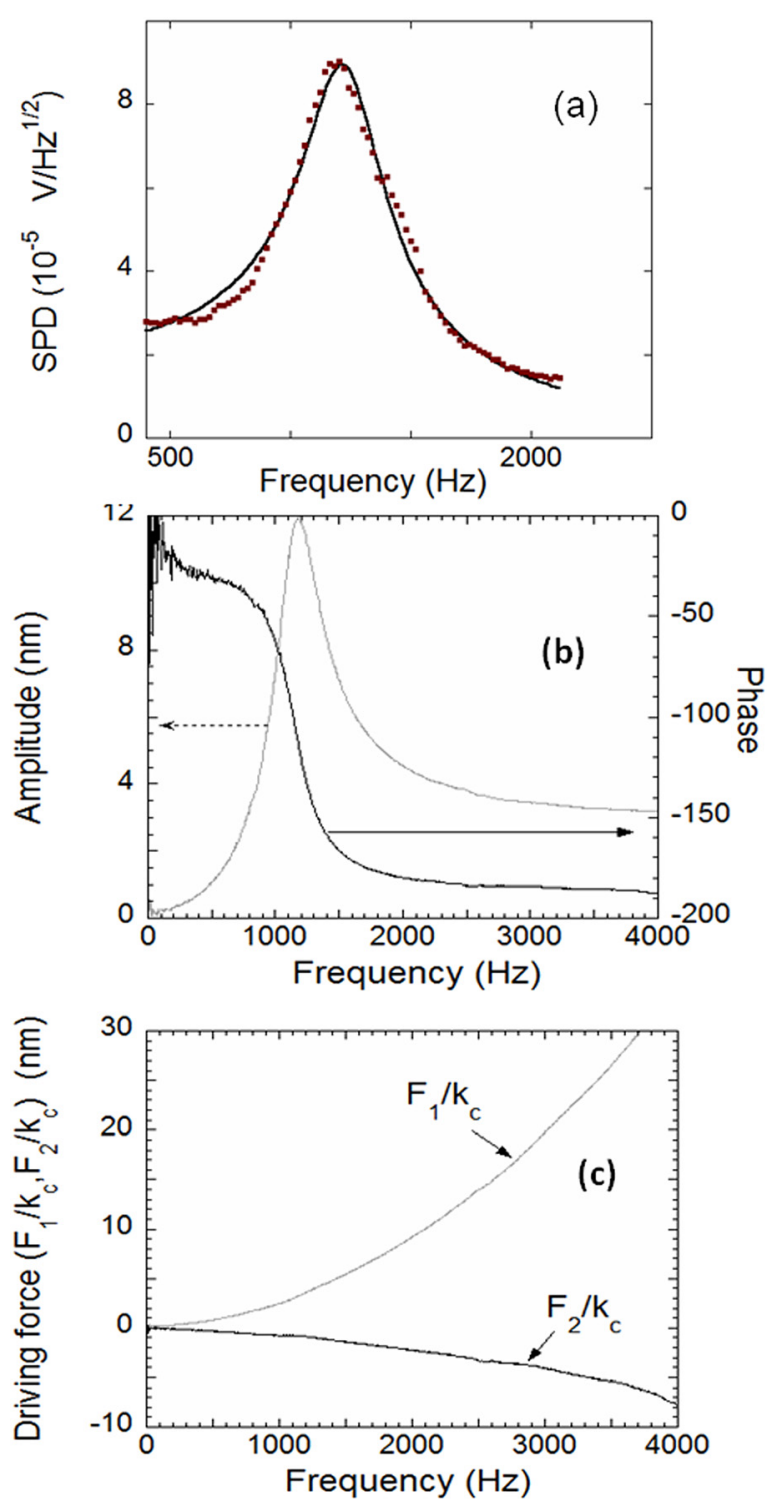

FIG. 4. (a) Thermal response of the cantilever in water. Continuous line is fitting curves using a simple damped harmonic oscillator model. (b) Resonance curve (amplitude and phase) measured by acoustic excitation. (c) The experimental driving forces that act on the cantilever extracted from the resonance curve.

infinite, and thus as expected from Eq. (5) $\left(A=A_{b}\right.$ when $\gamma_{\text {int }} \rightarrow \infty$ ), the measured cantilever deflection amplitude for all frequencies is equal to the base vibration amplitude. The amplitude of the base vibration was determined in this experiment for the vibrating frequencies 100, 200, and $1000 \mathrm{~Hz}$ to be $1.86,2.04$, and $2.06 \mathrm{~nm}$, respectively.

After measuring the driving force, the quality factor $Q$, the resonance frequency $\omega_{0}$, and the base amplitude $A_{b}$, we can apply Eq. (7a) to extract the interaction damping versus the distance from the measured amplitude and phase.

Fig. 6 shows the damping $\gamma_{\text {int }}$ versus the distance $D$ for different frequencies extracted from the data of the amplitude and phase plotted in Fig. 5(a) and Fig. 5(b). Note here that damping extracted for different vibration frequencies coincide with each other. We have also reported on this
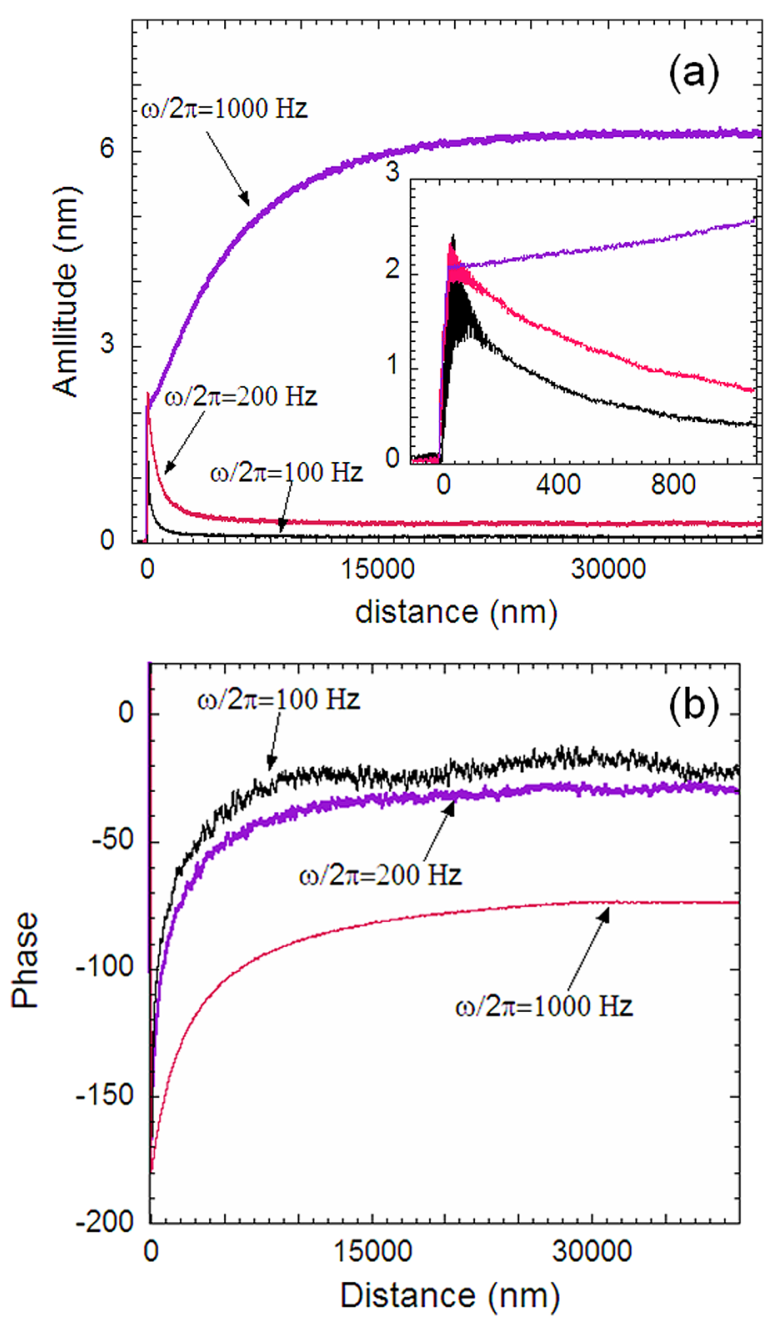

FIG. 5. (a) Cantilever amplitude versus the distance for different frequencies of the base vibration. The inset shows a zoom close to the contact position (distance smaller than $1000 \mathrm{~nm}$ ). (b) Phase versus distance.

figure, the hydrodynamic drag coefficient $\gamma_{h}=\frac{F_{h}}{V}$ measured using the contact mode. As expected, the drag coefficient $\gamma_{h}$ measured with a contact mode coincides with the damping coefficient $\gamma_{\text {int }}$ measured with the dynamic mode.

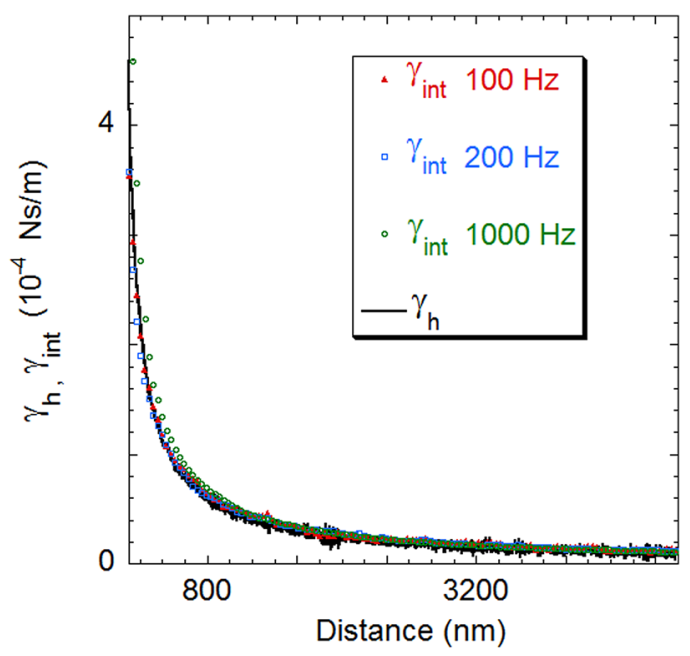

FIG. 6. Hydrodynamic drag coefficient $\gamma_{h}$ measured with a contact mode (dark continuous line) and the interaction damping coefficient $\gamma_{\text {int }}$ measured with the dynamic mode for different frequencies of excitation. 


\section{CONCLUSION}

In summary, we have shown how to extract accurately the acoustic driving force acting on the cantilever in liquid from the measured resonance curve. We have presented a model that includes the driving force to extract the damping and the stiffness of the tip sample interaction. Finally, experimental measurements were performed to validate the model.

The three steps of our method are recalled below

(1) Measure the quality factor and resonance frequency from the thermal response of the cantilever (actually, this operation is available in most new AFMs).

(2) Measure the acoustic driven resonance spectra of the cantilever and then extract the driven force using Eqs. (4a) and (4b).

(3) Use Eqs. (7a) and (7b) to convert amplitude and phase data to damping and stiffness.

\section{ACKNOWLEDGMENTS}

The authors thank G. Couturier for reading the manuscript and for helpful discussions. This work was supported by the French National Agency of research (MicRheo Project No. ANR-08-NANO-004) and by the Conseil Régional d'Aquitaine (No. 20091102001).

\section{APPENDIX: CONTRIBUTION OF THE ADDITIONAL FLUID ADDED MASS AND ADDED DAMPING CLOSE TO THE SURFACE}

During the interaction to describe the cantilever motion, we have to add to the second term of Eq. (1), all the contributions due to the interaction.

The equation of motion can be written as

$$
\begin{aligned}
m^{*} \ddot{X}+\gamma_{\text {bulk }} \dot{X}+k_{c} X= & \left(F_{1}+j F_{2}\right) e^{j \omega t}-k_{\text {int }}^{\prime} Z-\gamma_{\text {int }}^{\prime} \dot{Z} \\
& -\gamma_{\text {iadd }} \dot{Z}-m_{\text {add }}^{*},
\end{aligned}
$$

where $k_{\text {int }}^{\prime}, \gamma_{\text {int }}^{\prime}$ are, respectively, the interaction stiffness and damping due to pure tip-sample interaction and $m_{\text {add }}^{*}, \gamma_{\text {add }}$, are the additional fluid mass and damping due to the cantilever beam vibration close to the surface. Using the expressions of the tip deflection $X(t)=A e^{j(\omega t+\varphi)}$ and total tip displacement $Z=X(t)+A_{b} e^{j \omega t}$, we get

$$
\begin{aligned}
& -k_{\text {int }}^{\prime} Z-\gamma_{\text {int }}^{\prime} \dot{Z}-\gamma_{\text {iadd }} \dot{Z}-m_{\text {add }}^{*} \ddot{Z} \\
& \quad=-\left(k_{\text {int }}^{\prime}-m_{\text {add }}^{*} \omega^{2}\right) Z-\left(\gamma_{\text {int }}^{\prime}+\gamma_{\text {iadd }}\right) \dot{Z}=-k_{\text {int }} Z-\gamma_{\text {int }} \ddot{Z} .
\end{aligned}
$$

With $k_{\text {int }}=k_{\text {int }}^{\prime}-m_{\text {add }}^{*} \omega^{2}$ and $\gamma_{\text {int }}=\gamma_{\text {int }}^{\prime}+\gamma_{\text {add }}$. By substituting Eqs. (A2) in (A1), we get Eq. (5) of the text

$$
\begin{aligned}
& m^{*} \ddot{X}+\left(\gamma_{\text {bulk }}+\gamma_{\text {int }}\right) \dot{X}+\left(k_{c}+k_{\text {int }}\right) X \\
& \quad=\left(F_{1}+j F_{2}\right) e^{j \omega t}-\left(k_{\text {int }}+j \omega \gamma_{\text {int }}\right) A_{b} e^{j \omega t} .
\end{aligned}
$$

${ }^{1}$ G. Binnig, C. F. Quate, and C. Gerber, Phys. Rev. Lett. 56, 930 (1986).

${ }^{2}$ Y. Martin, C. C. Williams, and H. K. Wickramasinghe, J. Appl. Phys. 61, 4723 (1987).

${ }^{3}$ F. Ohnesorge and G. Binnig, Science 260, 1451 (1993).

${ }^{4}$ J. F. Giessibl, Science 267, 68 (1995).

${ }^{5}$ F. A. Schabert, C. Henn, and A. Engel, Science 268, 92 (1995).

${ }^{6}$ B. Cappella and G. Dietler, Surf. Sci. Rep. 34, 1 (1999).

${ }^{7}$ H. J. Butt, B. Cappella, and M. Kappl, Surf. Sci. Rep. 59, 1 (2005).

${ }^{8}$ R. Garcia and P. Perez, Surf. Sci. Rep. 47, 197 (2002).

${ }^{9}$ M. Lantz, Y. Z. Liu, X. D. Cui, H. Tokumoto, and S. M. Lindsay, Surf. Interface Anal. 27, 354 (1999).

${ }^{10}$ C. Jai, T. Cohen Bouhacina, and A. Maali, Appl. Phys. Lett. 90, 113512 (2007).

${ }^{11}$ E. T. Herruzo and R. Garcia, Appl. Phys. Lett. 91(14), 143113 (2007).

${ }^{12}$ X. Xu and A. Raman, J. Appl. Phys. 102, 034303 (2007).

${ }^{13}$ S. D. Beer, D. Van Den Ende, and F. Mugele, Appl. Phys. Lett. 93(25), 253106 (2008).

${ }^{14}$ D. Kiracofe and A. Raman, Nanotechnology 22(48), 485502 (2011).

${ }^{15}$ R. G. Horn, O. I. Vinogradova, M. E. Mackay, and N. Phan Thien, J. Chem. Phys. 112, 6424 (2000).

${ }^{16}$ C. D. Honig and W. A. Ducker, Phys. Rev. Lett. 98, 028305 (2007).

${ }^{17}$ L. Zhu, P. Attard, and C. Neto, Langmuir 27, 6712 (2011). 